\title{
Mobile Phones of 3G Era in Small and Medium-Sized Agricultural Production and Application Prospect
}

\author{
Yang Yang, Tian He, and Yang Zhang \\ School of Mechanical and Electronical Engineering, East China Jiaotong University, \\ Nanchang, Jiangxi, China \\ hetian1985@yahoo.cn
}

\begin{abstract}
The arrival of the era of third-generation (3G) mobile phone will cause earth-shaking changes, as a kind of new communication technologies will play in the fields of estimation. The rapid development of electronic technology and information technology, which had brought about a revolution of control and management of agricultural, mobile $3 \mathrm{G}$ era will give the agricultural revolution fueled, and bring a new era of agricultural productivity liberation. Mobile phones of $3 \mathrm{G}$ can achieve the real-time monitoring and control of agricultural through automatic control and electronic computer technology and communication technology, but also can get rid of the remote control and regional restrictions. Mobile phones of $3 \mathrm{G}$ realize the agricultural field of remote real-time automatic control through video image processing and fast speed of technology.
\end{abstract}

Keywords: mobile phones of $3 \mathrm{G}$ agricultural production automation medium and small enterprises.

\section{Introduction}

China is a large agricultural country, but the level of agricultural production is still in the hand-cultivation, not to achieve a large area of mechanized mass production situation. It is required a considerable period of time that achieve large-scale mechanized production because of China's economic system and the geographical features. Therefore based on China's national conditions, the development of small-scale automated production should be present a fresh development trend of agricultural production which difficult to implement joint large-scale operation situation because of Contracting by an individual. Our agricultural production may be contracted to individuals which results in a region the land under the area of fragmented, together with mountainous and hilly region, which brings large-scale machinery to some trouble. Combined with China's national conditions and characteristics of China's agricultural production patterns, it is necessary to achieve the implementation of small-scale automated production of Chinese in agricultural machinery automation.

Along with fast development and compact combination of both electronics technology and communication technology ,a person may be have more than a cell phone in average .With entered into an age of $3 \mathrm{G}$, using a mobile phone deal with information is the exception ordinary daily life. Mobile communication terminal as a microprocessor controlled intelligent sensor automatic production mode. 
$3 \mathrm{G}$, full name of the third Generation. It enhanced voice and data in transmission speed based on previous data. It can be better in the global seamless roaming, and process images, music, video streaming and other forms of media, provided various services including web browsing, conference calls, e-commerce and other information services. Wireless network can support different data transfer rate, for example at least were 2Mbps (megabits / second), 384kbps (kilobits / second) and 144kbps transmission speed indoor, outdoor, and driving environment. With high-speed $3 \mathrm{G}$ network to download opens up a new era of mobile multimedia, mobile phone is bound to be another PC.

As rapid development of electronic information and communication technology, agriculture has been basically achieve modernization and automation. using of $3 \mathrm{G}$ mobile phone and electronic information and communications technology to simplify and automatic agricultural control, agricultural production must be brought to the rapid leap. 3G mobile phone technology as the core of the control will adapt to the scale of agricultural production, promote small and medium agricultural production in China, show the extraordinary in all aspects of the function.

\section{Automated Irrigation Control}

60 years of New China, the Chinese effective irrigation area expanded from 2.4 million hectares to 8.77 million mu, accounting for the world's total 1 / 5, ranking first in the world. China to account for $48 \%$ of the irrigated land area, produces $75 \%$ of the country's total output of grain and $90 \%$ cotton, vegetables and other cash crops. At present the existing embankments, small pumping stations, motor-pumped wells, ponds, cellars and other small-scale water conservancy projects and the independent operation of more than two million, medium-sized irrigation channels to the end of class, small irrigation channels of nearly 3 million kilometers fixed, fixed at about 1.8 million kilometers of irrigation channels, the corresponding supporting structures close to 7 million, countless field projects cover nearly all of irrigated farmland.

Can see from the above data, our small-scale irrigation systems in a dominant position, to achieve small-scale irrigation which automatic irrigate and monitor is essential. As the motor-pumped wells and ponds occupy a vast cellar rate in the small-scale irrigation, Therefore, the establishment of $3 \mathrm{G}$ mobile phone system as the core of the automation of irrigation means which is a automation project of easy to be afforded and implemented. It is composed of the core of $3 \mathrm{G}$ mobile phone, Sensor data in realtime measurement of the field and the SCM system to accept delivery system consisting of wireless, The system limit the use of fuzzy criteria to achieve the simplification of small and medium irrigation, which meet the educational level of farmers limited. Using of the project to control pump motor starts and stops, irrigation canal gate opening and closing to achieve automatic irrigation of farmland, The function of System are real-time control, real-time data acquisition and preservation, on-site real-time video surveillance capabilities, unmanned operation of the field, which achieve realtime monitoring of the situation but no field operations. That is the advantage of $3 \mathrm{G}$ mobile phones has a strong transmission capacity, to ensure real-time agriculture and automation. 


\section{Livestock Breeding Automation}

In order to increase rural incomes, farmers increase the breeding livestock during agricultural production. The problems is Automatic feeding and automatic watering for farmers while agricultural production.

As the farmers economic conditions, small line of automation systems to be a star which build of sensor technology and $3 \mathrm{G}$ mobile communications technology, In recent years, with the prices of live pigs and breeding conditions of randomness, greenhouse pig become a new means of building up a fortune. Using $3 \mathrm{G}$ mobile phone integrated automation system, monitoring of pigs could be the case anywhere, automatic feeding, automatic watering according to real-time detection of ambient temperature and humidity, to adjust necessary conditions for pig. Automated system for $3 \mathrm{G}$ mobile phone adapt to individual small-scale farming in rural China, emancipate the productive forces, which accelerate the income of farmers, meet the basic requirements of the State.

\section{Greenhouse Automation}

Greenhouse provides a new modern production environment to agriculture, and achieved good economic and social benefits. It can raise the income of countrymen, improve the industrial level and the ability to withstand natural disasters to extend the time for crop growth, increase crop yields, and so on. With the continuous development of the economy develop simultaneously on the greenhouse temperature, humidity, carbon dioxide and other automated monitoring and control has also been a corresponding development, thus improving income. using of electronic and information technology monitoring and control of greenhouse climate change data, such as temperature, humidity, illumination time and intensity, wind direction and wind speed and other factors, then back to the $3 \mathrm{G}$ mobile phone, to judge by artificial simple, once again sent through mobile phones order to plant growth requirements under the automatic adjustment. For example the temperature and humidity control. If the temperature is too low or too high, can be furnace heated up or can exhaust or otherwise deal with. If the humidity is too low, you can start the sprinkler system, when the humidity is too high can exhaust fan out of the air humidity is too high. At the same time can be $\mathrm{CO} 2$, light, irrigation and other automatic control. According to different crops, different times, $3 \mathrm{G}$ mobile phones to set different commands, using different control targets and control modes.

\section{Conclusion}

3G mobile phone communications technology combined with sensor technology to realize the automation of small and medium agricultural production, reduce labor input to achieve the implementation of the monitoring of real-time control and remote control can be achieved, without geographical restrictions, thereby bringing agricultural labor simplify the automation, improved economic efficiency, reducing labor. 


\section{References}

[1] Industrial Computer Application in agriculture

[2] Computer control and management of greenhouse technology development overview and prospects in China, Agriculture Computer Miscellaneous

[3] The Chinese Academy of Agricultural Irrigation Water Science and Technology Development Center, China Rural Water Network (2010) 